\title{
An Aggressive Plasmablastic Lymphoma of the Oral Cavity as Primary Manifestation of Acquired Immunodeficiency Syndrome: Case Report and Literature Review
}

\author{
${ }^{1}$ Department of HIV/AIDS, Infectious Diseases F. J. Muñiz Hospital, \\ Buenos Aires, Argentina \\ ${ }^{2}$ Department of Internal Medicine, University of Buenos Aires School \\ of Medicine, Paraguay, Buenos Aires, Argentina \\ ${ }^{3}$ Department of Hematology Unit, Infectious Diseases F. J. Muñiz \\ Hospital, Buenos Aires, Argentina \\ ${ }^{4}$ Histopathology Laboratory, Infectious Diseases F. J. Muñiz Hospital, \\ Buenos Aires, Argentina \\ ${ }^{5}$ Sociedad Iberoamericana de Información Científica, \\ Buenos Aires, Argentina
}

Marcelo Corti ${ }^{1,2}$ Gonzalo Minué ${ }^{3}$ Ana Campitelli ${ }^{4}$ Marina Narbaitz ${ }^{4}$ Leonardo Gilardi ${ }^{5}$

Int Arch Otorhinolaryngol 2015;19:354-358.

Address for correspondence Marcelo Corti, MD, Department of HIV/AIDS, Infectious Diseases F. J. Muñiz Hospital, Puán $3812^{\circ}$ floor, Buenos Aires C1406CQG, Argentina (e-mail: marcelocorti@fibertel.com.ar).

\author{
Abstract \\ Keywords \\ - plasmablastic \\ lymphoma \\ - oral cavity \\ - HIV \\ - AIDS
}

Introduction Plasmablastic lymphoma is a rare entity that was first described in the jaws and the oral cavity of patients with human immunodeficiency virus (HIV) and acquired immunodeficiency syndrome (AIDS). Plasmablastic lymphoma is considered as a diffuse, large, B-cell lymphoma with a unique phenotype and a predilection for the oral cavity. Objective The authors describe a case of an aggressive plasmablastic lymphoma of the oral cavity as the primary manifestation of AIDS.

Resumed Report We report a case of plasmablastic lymphoma involving only the oral cavity as the first manifestation of AIDS. Diagnosis was confirmed by the oral lesion biopsy and the histopathologic examination that showed a dense infiltrate composed of atypical lymphocytes with numerous plasmocytes that expressed the plasma cell markers MUM-1 and CD138 and that were negative for the B-cell markers CD3, CD20, and CD45. Immunohistochemical and in situ hybridization revealed the Epstein-Barr virus genome in the atypical cells. Polymerase chain reaction was also positive for human herpesvirus-8 RNA.

Conclusion The HIV serologic status should be evaluated in all patients with plasmablastic lymphoma of the oral cavity or extraoral sites.

\section{Introduction}

Non-Hodgkin's lymphoma (NHL) is the second most frequent malignancy in patients with acquired immunodeficiency syndrome (AIDS). More than $90 \%$ of human immunodeficiency virus (HIV)-associated NHL is derived from B cells and the majority is of high grade. Extranodal presentation is more common in HIVseropositive patients and occurs in 70 to $80 \%$ of cases. ${ }^{1,2}$

The oral cavity is a rare site of presentation for $\mathrm{NHL}$, and only $3 \%$ of them involve primarily the oral cavity. ${ }^{3}$ Plasmablastic lymphoma (PBL) is an aggressive subtype of diffuse large B-cell lymphoma (DLBCL) that is diagnosed more received

August 21, 2014

accepted

November 14, 2014

published online

January 8, 2015
DOI http://dx.doi.org/

10.1055/s-0034-1397335. ISSN $1809-9777$.
Copyright $\odot 2015$ by Thieme Publicações License terms

Ltda, Rio de Janeiro, Brazil
(ब) $\Theta \circledast$ 
frequently in patients infected with HIV. ${ }^{4}$ HIV-associated PBL is associated with an aggressive clinical course, poor prognosis, and short survival even in the era of highly active antiretroviral therapy (HAART). ${ }^{5,6}$

We report a patient who developed an aggressive oral PBL as primary manifestation of AIDS.

\section{Review of the Literature with Differential Diagnosis}

PBL was first described in HIV-seropositive subjects by Delecluse et al and typically involves the oral cavity, ${ }^{4}$ especially the jaw and the palate, and accounts for $2.6 \%$ of all NHLs in this population. ${ }^{7}$ However, this rare neoplasm has been described in other sites such as lymph nodes, subcutaneous soft tissue, liver, bones, and anorectum. ${ }^{8} \mathrm{~A}$ small number of cases have been reported in the medical literature, with the majority being case reports or small case series. ${ }^{9}$ Ninety-two percent of oral PBL cases described in the medical literature were patients infected with HIV. ${ }^{10,11}$

Besides the PBLs, other subtypes of B-cell NHLs may involve the oral cavity, including DLBCL and the Burkitt lymphoma. The differential diagnosis of large oral tumor lesions in patients with AIDs includes infections (intraoral tissue abscesses) and other malignant clinical entities, different from NHL. Infectious diseases that can involve the oral cavity include odontogenic abscesses caused by the oral microflora bacteria and oral lesions caused by fungi, especially Histoplasma capsulatum. Malignancies include Kaposi sarcoma of the oral cavity as initial and sole manifestation or associated with other mucocutaneous lesions, primary squamous cell carcinoma related with human papillomavirus infection, and metastatic lesions.

\section{Case Report}

A 39-year-old man with a history of intravenous drug abuse was admitted to our HIV/AIDS Department with a 1-month history of mild fever, weight loss, and night sweats. He presented with oral pain and odynophagia and dysphagia. On physical examination, the only remarkable finding was a large, nonfluctuant, and ulcerative mass, including dental pieces, with areas of necrosis, which involved the hard palate and the gingiva with a painful swelling over the right upper jaw. The lesion increased rapidly in size within the previous month (-Figs. 1 and 2). No peripheral cervical adenopathies were detected. Significant laboratory findings showed hemoglobin $11.30 \mathrm{~g} \%$, hematocrit $34 \%$, white blood cells $3,300 / \mathrm{mm}^{3}$, platelets $39,000 / \mathrm{mm}^{3}$, erythrocyte sedimentation rate $78 \mathrm{~mm} / \mathrm{h}$, lactate dehydrogenase (LDH) $662 \mathrm{U} / \mathrm{L}$, and alkaline phosphatase $130 \mathrm{IU} / \mathrm{L}$. Renal and liver function were normal. A rapid enzymelinked immunosorbent assay (ELISA) for HIV was positive; HIV diagnosis was confirmed by fourth-generation ELISA and Western blot. Hepatitis $C$ antibodies were also positive. The CD4 T-lymphocyte count was 57 cells $/ \mu \mathrm{L}(8 \%)$ and the plasma viral load was 93,557 copies/mL $\log _{10} 5.0$.

A computed tomography (CT) scan of the maxillofacial area revealed a large and heterogeneous mass with areas of necrosis

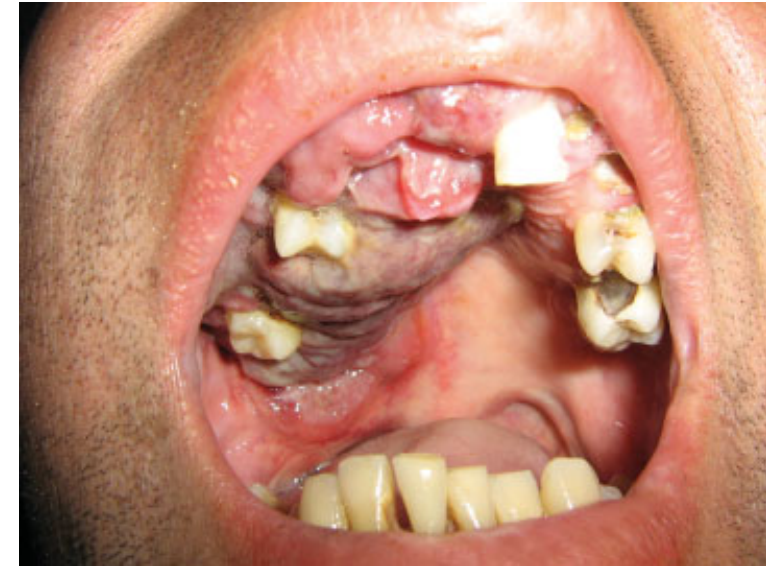

Fig. 1 Initial clinical appearance: a large, nonfluctuant, necrotizing, and ulcerated gingival lesion located at the right upper jaw, the gingiva, and the palate.

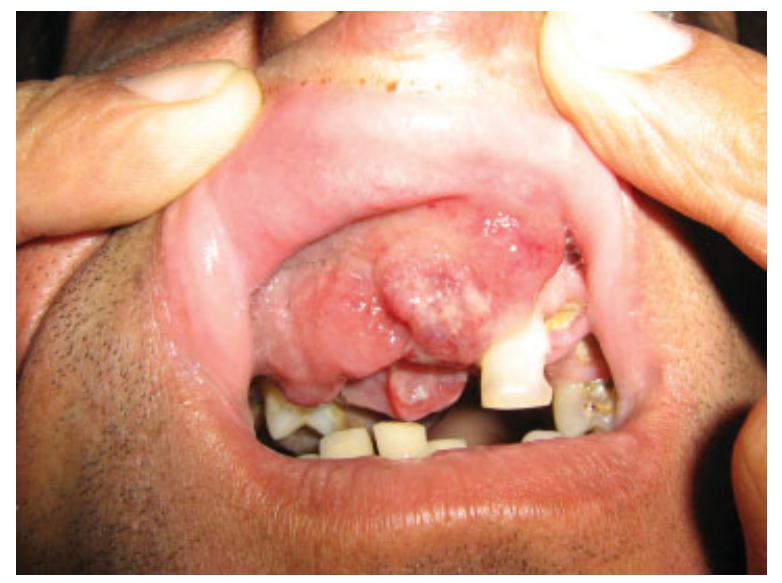

Fig. 2 The large initial lesion including dental pieces at the right upper jaw, with infiltration of the palate and the gingiva.

involving the upper right gingival and the right hard palate with bone erosion of the upper jaw (-Figs. 3 and $\mathbf{4}$ ). A presumptive diagnosis of primary NHL of the oral cavity associated with AIDS was made and an incisional biopsy was performed.

The histopathologic examination of the biopsy smears showed a diffuse proliferation of atypical lymphoid cells with extensive areas of necrosis of the oral mucosa. The atypical cells contained large polygonal plasmablastic cells with abundant basophilic cytoplasm and large and excentric nuclei with a prominent central nucleoli associated with marked mitotic and apoptotic findings ( - Fig. 5). Immunohistochemical examination revealed that the majority of tumor cells expressed the plasma cells markers CD138, VS38c, and MUM- 1 and were negative for $\mathrm{CD} 3, \mathrm{CD} 20, \mathrm{CD} 45, \mathrm{Bcl} 2$, and $\mathrm{Bcl} 6$ (-Fig. 6). The Ki67 index was nearly $80 \%$, revealing a high proliferation rate. A bone marrow biopsy was negative for neoplastic infiltration. A CT scan of the chest, abdomen, and pelvis did not show any abnormal findings. The histologic morphology and the plasma cell immunophenotype established the final diagnosis of primary PBL of the oral cavity. 


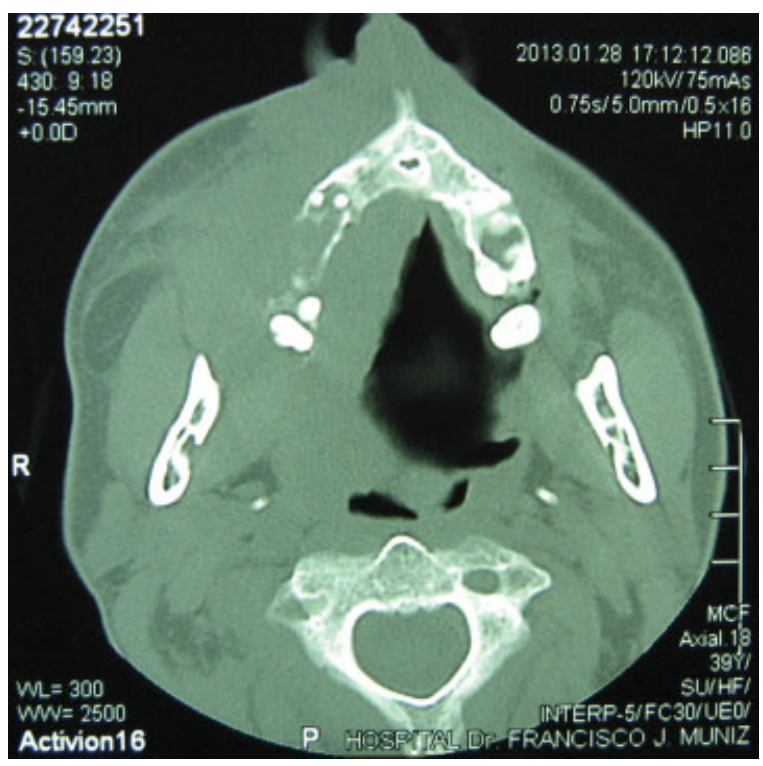

Fig. 3 Axial computed tomography scan of the head and face showing a large and heterogeneous mass with areas of necrosis and a partial occupation of the cavum.

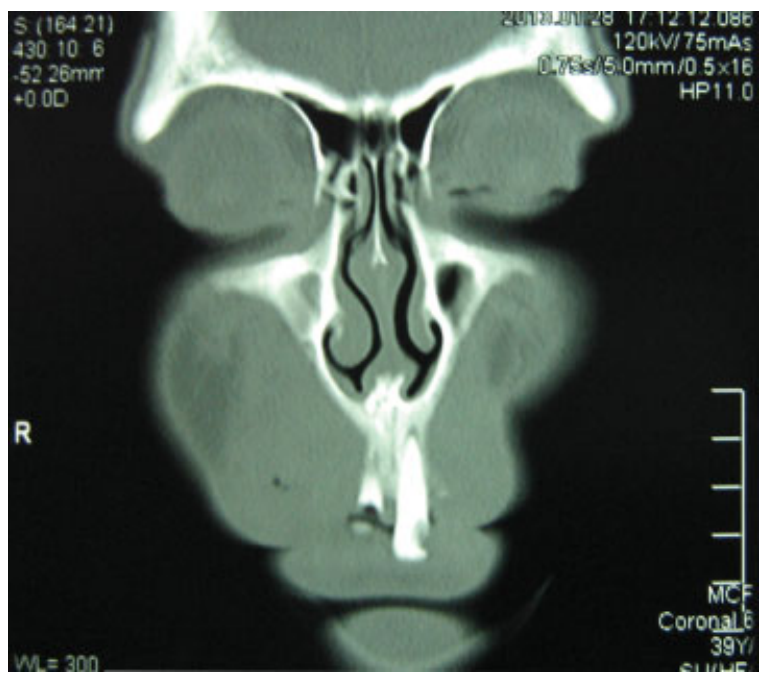

Fig. 4 Coronal computed tomography scan of the maxillofacial region revealed a large mass with areas of necrosis involving the upper right gingival and the right hard palate with bone erosion of the upper jaw.

The Epstein-Barr (EBV) virus genome was detected in the atypical tumor cells by in situ hybridization (ISH; - Fig. 7). Polymerase chain reaction (PCR) was positive for human herpes virus 8 (HHV-8) RNA in the tumor cells.

After a partial response to the first cycle of chemotherapy based on CHOP (cyclophosphamide, vincristine, doxorubicin, and prednisolone) plus preventive intrathecal chemotherapy (methotrexate and cytarabine) plus HAART (abacavir plus lamivudine plus efavirenz), the neoplasm relapsed, with a large, ulcerative, and necrotizing lesion that involved both hard and soft palate, the gingiva, and the upper maxilla and that took up the oral cavity. The lesion did not respond to the two following cycles of chemotherapy. Two months later, the

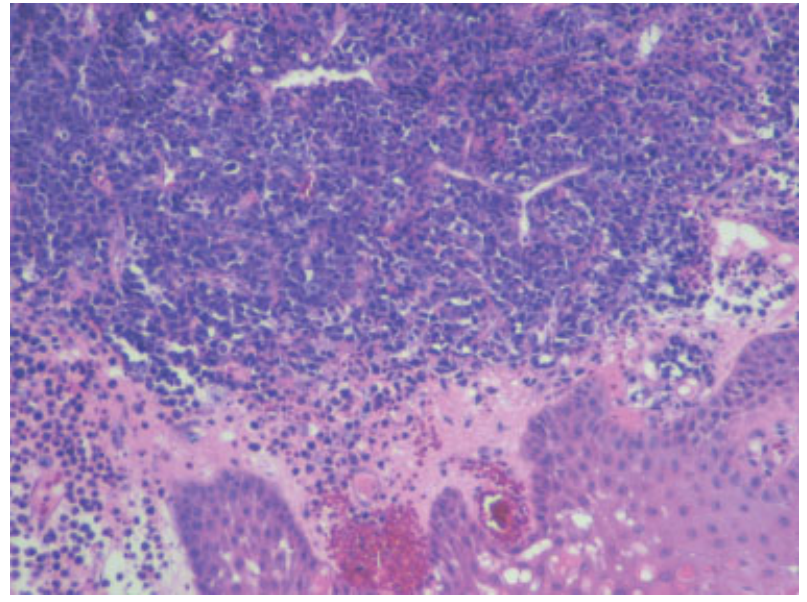

Fig. 5 Oral mucosal lesion biopsy revealed the existence of an atypical and dense infiltrate, relativity uniform, composed of large cells with a moderate cytoplasm, an eccentric nuclei, and one or more large nucleoli. Also, it is possible to see a variable number of small cells with plasmacytic appearance, consistent with the diagnosis of high-grade non-Hodgkin lymphoma. Hematoxylin and eosin, $\times 200$.

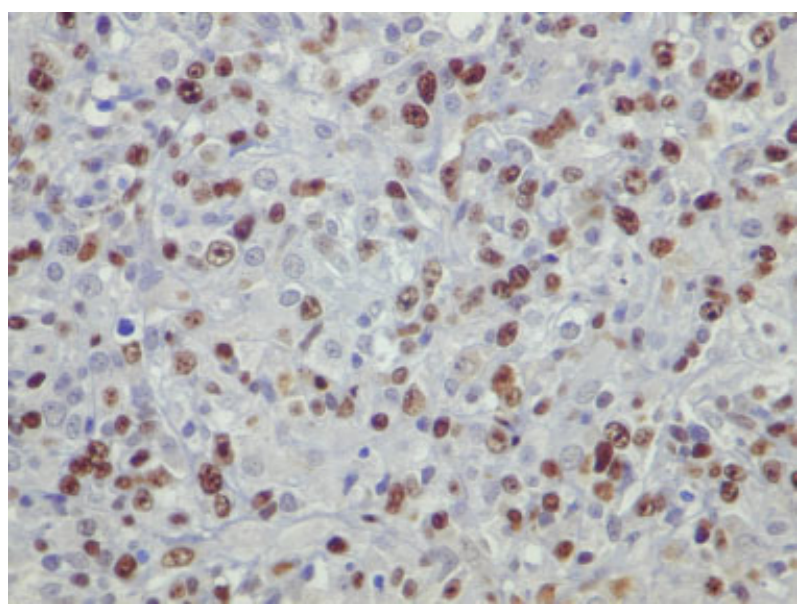

Fig. 6 Immunohistochemical phenotype of plasmablastic lymphoma: the majority of cells expressed the B cell antigens CD138 and MUM1.

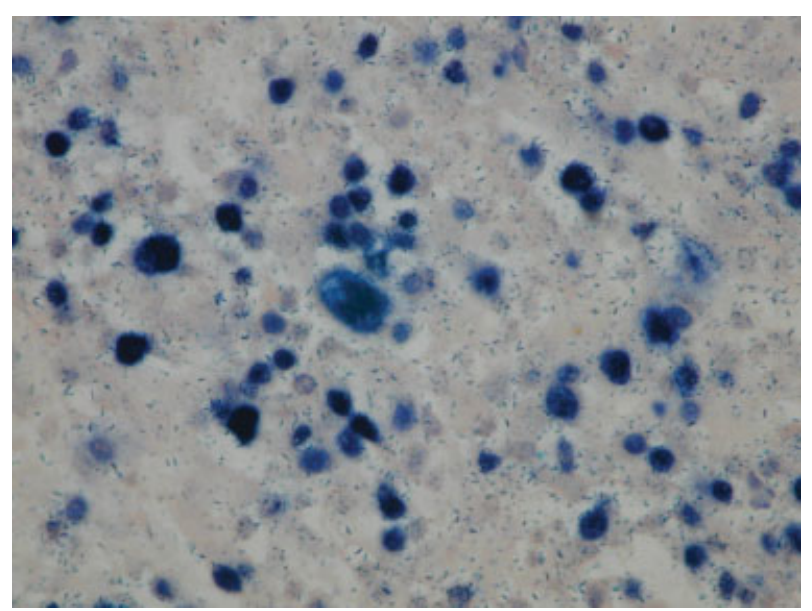

Fig. 7 In situ hybridization: Epstein-Barr virus-encoded RNA (EBV genome) was expressed with monomorphic cytologic features in the atypical cells. 
patient died due to the lymphoma progression and sepsis with multiorgan failure.

\section{Discussion}

Patient with HIV infection are at increased risk to develop NHL. These lymphomas are characterized by rapid progression, frequent extranodal initial manifestation, and a poor outcome. ${ }^{5,6}$

PBL was first described in HIV-seropositive subjects by Delecluse et $\mathrm{al}^{4}{ }^{4}$ and it typically involves the oral cavity, especially the jaw and the palate, and accounts for $2.6 \%$ of all NHLs in this population. ${ }^{7}$ However, this rare neoplasm has been described in other sites such as lymph nodes, subcutaneous soft tissue, liver, bones, and anorectum. ${ }^{8} \mathrm{~A}$ small number of cases have been reported in the medical literature, with the majority as case reports or small case series. ${ }^{9}$ Ninetytwo percent of oral PBL cases described in the medical literature were HIV-infected patients. ${ }^{10,11}$

PBL is included in the World Health Organization classification as a subtype of lymphoma described most frequently in HIV-infected patients. ${ }^{6}$ This lymphoma is characterized by an unusual immunophenotype with negative CD20 and a predominant plasma cell differentiation, expressing markers such as CD38, CD138, and MUM1 protein. PBL immunophenotype is similar to myeloma; however, using genomic profiling, PBL seems closer to DLBCL. ${ }^{12}$

Clinically, the most significant characteristic of PBL in patients with AIDS is the compromise of the oral cavity, including the hard palate, the gingiva, and the jaw, as occurred in our patient, who had large masses as the unique clinical finding. Gingival involvement is characteristic in HIVseropositive patients, whereas the tongue involvement is more frequent in immunocompetent patients. PBL of the oral cavity always involves the hard palate. ${ }^{13}$

Histopathologic characteristics of PBL include the advanced stage of the neoplasm at diagnosis, elevated LDH levels, and a very high proliferation index (Ki67 level) more than $80 \%$, as we saw in our patient. ${ }^{5}$ Histologic differential diagnosis of oral PBL includes Burkitt lymphoma of the oral cavity with plasmablastic differentiation, anaplastic subtypes of DLBCL, and poorly differentiated oral carcinomas. ${ }^{5}$

EBV is strongly associated with the pathogenesis of PBL in patients with AIDS, and EBV expression by atypical cells is frequent in AIDS-associated PBL (70\% of cases). ${ }^{14}$ The presence of EBV genome can be detected in atypical cells by two techniques, immunohistochemistry for latent membrane protein-1 and ISH for Epstein-Barr encoding region (EBERs-1 RNA). Additionally, detection of HHV-8 DNA was also positive by PCR in our patient.

PBL in patients with AIDS is generally associated with a poor prognosis. The most common causes for this poor prognosis include the regular relapsing evolution of PBL, which can be explained by the severe immunosuppression associated with advanced HIV/AIDS disease, and the chemoresistance of PBL, which explains the most frequent cause of death (lymphoma progression, as we observed in our patient). Development of infectious complications is the second most common cause of death in these patients. ${ }^{11,13}$
Treatment of PBL is similar to other aggressive lymphomas; CHOP or CHOP-like regimens are the most common therapeutic alternatives. Although some physicians use regimens more intensive than CHOP, the role of such therapy is unclear. $^{5,12}$

Castillo et al analyzed retrospectively 70 patients with HIV-associated PBL treated with chemotherapy. ${ }^{5}$ In this series, the mean $\mathrm{CD} 4{ }^{+}$T-cell count was 165 cells $/ \mu \mathrm{L}$, and the overall response rate to chemotherapy was $77 \%$, but only $46 \%$ of the patients presented complete response. The overall survival rate was 14 months, and $72 \%$ of patients died from lymphoma progression, as in our case. In this study, the use of regimens more intensive than $\mathrm{CHOP}$ were not associated with a better survival.

\section{Final Comments}

PBL is an aggressive subtype of DLBCL with distinct histopathologic features, pathogenic viral associations, and outcome. The association between PBL and HIV infection has been well demonstrated, with a high prevalence in the oral cavity. ${ }^{8,9,15}$

In summary, PBL should be included in the differential diagnosis of large masses involving the oral cavity. The HIV serologic status should be evaluated in all patients with PBL of the oral cavity or extraoral sites.

\section{References}

1 Levine AM. Acquired immunodeficiency syndrome-related lymphoma. Blood 1992;80(1):8-20

2 Gandhi MK, Khanna R. Viruses and lymphoma. Pathology 2005; 37(6):420-433

3 Takahashi H, Fujita S, Okabe H, Tsuda N, Tezuka F. Immunophenotypic analysis of extranodal non-Hodgkin's lymphomas in the oral cavity. Pathol Res Pract 1993;189(3):300-311

4 Delecluse HJ, Anagnostopoulos I, Dallenbach F, et al. Plasmablastic lymphomas of the oral cavity: a new entity associated with the human immunodeficiency virus infection. Blood 1997;89(4): 1413-1420

5 Castillo JJ, Furman M, Beltrán BE, et al. Human immunodeficiency virus-associated plasmablastic lymphoma: poor prognosis in the era of highly active antiretroviral therapy. Cancer 2012;118(21): 5270-5277

6 Tirelli U, Spina M, Gaidano G, Vaccher E, Franceschi S, Carbone A. Epidemiological, biological and clinical features of HIV-related lymphomas in the era of highly active antiretroviral therapy. AIDS 2000;14(12):1675-1688

7 Hansra D, Montague N, Stefanovic A, et al. Oral and extraoral plasmablastic lymphoma: similarities and differences in clinicopathologic characteristics. Am J Clin Pathol 2010;134(5):710-719

8 Corti M, Villafañe MF, Bistmans A, Campitelli A, Narbaitz M, Baré P. Oral cavity and extra-oral plasmablastic lymphomas in AIDS patients: report of five cases and review of the literature. Int $\mathrm{J}$ STD AIDS 2011;22(12):759-763

9 Rafaniello Raviele P, Pruneri G, Maiorano E. Plasmablastic lymphoma: a review. Oral Dis 2009;15(1):38-45

10 Chang CC, Zhou X, Taylor JJ, et al. Genomic profiling of plasmablastic lymphoma using array comparative genomic hybridization (aCGH): revealing significant overlapping genomic lesions with diffuse large B-cell lymphoma. J Hematol Oncol 2009;2:47

11 Sarode SC, Zarkar GA, Desai RS, Sabane VS, Kulkarni MA. Plasmablastic lymphoma of the oral cavity in an HIV-positive patient: a 
Corti et al.

case report and review of literature. Int J Oral Maxillofac Surg 2009;38(9):993-999

12 Tsachouridou O, Christoforidou A, Metallidis S, et al. Plasmablastic lymphoma of the oral cavity, a B cell-derived lymphoma associated with HIV infection: a case series. Eur Arch Otorhinolaryngol 2012; 269(6):1713-1719

13 Cattaneo C, Facchetti F, Re A, et al. Oral cavity lymphomas in immunocompetent and human immunodeficiency virus infected patients. Leuk Lymphoma 2005;46(1):77-81
14 Hamilton-Dutoit SJ, Raphael M, Audouin J, et al. In situ demonstration of Epstein-Barr virus small RNAs (EBER 1) in acquired immunodeficiency syndrome-related lymphomas: correlation with tumor morphology and primary site. Blood 1993;82(2): 619-624

15 Colomo L, Loong F, Rives S, et al. Diffuse large B-cell lymphomas with plasmablastic differentiation represent a heterogeneous group of disease entities. Am J Surg Pathol 2004;28(6): 736-747 